\title{
In vivo hyperspectral analysis of skin hemoglobin and melanin content for neoplasia detection
}

\author{
Ivan A. Bratchenko ${ }^{1 *}$, Oleg O. Myakinin ${ }^{1}$, Violetta P. Sherendak ${ }^{1}$, Pavel N. Volkhin ${ }^{1}$, \\ Yulia A. Khristoforova ${ }^{1}$, Lyudmila A. Bratchenko ${ }^{1}$, Dmitry N. Artemyev ${ }^{1}$, Alexander A. Moryatov ${ }^{2}$, \\ Olga V. Polschikova ${ }^{3}$, Alexander S. Machikhin ${ }^{3}$, Vitold E. Pozhar ${ }^{3}$, Sergey V. Kozlov², \\ and Valery P. Zakharov ${ }^{1}$ \\ ${ }^{1}$ Department of Laser and Biotechnical Systems, Samara National Research University, 34 Moskovskoye shosse, \\ Samara 443086, Russia \\ ${ }^{2}$ Department of Oncology, Samara State Medical University, 80 Polevaya Street, Samara 443099, Russia \\ ${ }^{3}$ Scientific and Technological Center of Unique Instrumentation, RAS, 15 Butlerova Street, Moscow 117342, Russia \\ * e-mail: iabratchenko@gmail.com
}

\begin{abstract}
We present results of main skin chromophores (melanin and hemoglobin) optical analysis. Analysis of 91 in vivo skin tissues (50 benign and 41 malignant) was performed in visible spectral region with hyperspectral imaging technique. To assess the malignancy of skin tissues we proposed two methods for calculating the integral index of tissue optical density and performed a comparison of their effectiveness and effectiveness of physician survey. As the main diagnostic feature, we propose to use data of integral optical index dispersion from the studied tissue and healthy tissue area. The results of skin tissues classification with discriminant analysis are presented. The possibility of the proposed approaches application in the clinical practice is shown. (C) 2018 Journal of Biomedical Photonics \& Engineering.
\end{abstract}

Keywords: Hyperspectral imaging; skin cancer; melanoma; basal cell carcinoma; skin chromophores.

Paper \#3310 received 17 Oct 2018; revised manuscript received 12 Dec 2018; accepted for publication 20 Dec 2018; published online 31 Dec 2018. doi: 10.18287/JBPE18.04.040301.

\section{References}

1. F. Bray, M. Colombet, L. Mery, M. Piñeros, A. Znaor, R. Zanetti, and J. Ferlay, Cancer Incidence in Five Continents. Vol. XI, International Agency for Research on Cancer, Lyon (2017).

2. R. Siegel, D. Nai-shadham, and A. Jemal, "Cancer statistics," CA: A Cancer Journal for Clinicians 62(1), 1029 (2012).

3. P. Boyle, D. Parkin, World Cancer Report 2008, International Agency for Research on Cancer, Lyon (2008).

4. A. D. Kaprin, V. V. Starinsky, and G. V. Petrova, Malignant neoplasms in Russia in 2015 (morbidity and mortality), P. A. Hertsen Moscow Oncology Research Center, Moscow (2017).

5. P. E. Gross, K. Strasser-Weippl, B. L. Lee-Bychkovsky, et al., "Challenges to effective cancer control in China, India, and Russia," The Lancet Oncology 15(5), 489-538 (2014).

6. C. B. L. M. Majoie, F.-J. H. Hulsmans, J. A. Castelijns, A. Walter, J. Bras, and F. L. M. Peeters, "Perineural tumor extension of facial malignant melanoma: CT and MRI," Journal of Computer Assisted Tomography 17(6), 973-975 (1993).

7. G. Argenziano, H. P. Soyer, "Dermoscopy of pigmented skin lesions - a valuable tool for early diagnosis of melanoma," The Lancet Oncology 2(7), 443-449 (2001).

8. I. A. Bratchenko, D. N. Artemyev, O. O. Myakinin, Y. A. Khristoforova, A. A. Moryatov, S. V. Kozlov, and V. P. Zakharov, "Combined Raman and autofluorescence ex vivo diagnostics of skin cancer in near-infrared and visible regions," Journal of Biomedical Optics 22(2), 027005 (2017). 
9. L. Lim, B. Nichols, M. Migden, N. Rajaram, J. Reichenberg, M. K. Markey, M. I. Ross, and J. W. Tunnell, "Clinical study of noninvasive in vivo melanoma and nonmelanoma skin cancers using multimodal spectral diagnosis," Journal of Biomedical Optics 19(11), 117003 (2014).

10. M. A. Calin, V. Sorin, D. Savastru, and M. Dragos, "Hyperspectral Imaging in the Medical Field: Present and Future," Applied Spectroscopy Reviews 49(6), 435-447 (2014).

11. F. Nachbar, W. Stolz, T. Merkle, A. B. Cognetta, T. Vogt, M. Landthaler, P. Bilek, O. Braun-Falco, and G. Plewig, "The ABCD rule of dermatoscopy. High prospective value in the diagnosis of doubtful melanocytic skin lesions," Journal of the American Academy of Dermatology 30(4), 551-559 (1994).

12. H. A. Haenssle, B. Korpas, C. Hansen-Hagge, T. Buhl, K. M. Kaune, A. Rosenberger, U. Krueger, M. P. Schön, and S. Emmert, "Seven-point checklist for dermatoscopy: performance during 10 years of prospective surveillance of patients at increased melanoma risk," Journal of the American Academy of Dermatology 62(5), 785-793 (2010).

13. P. Bourne, C. Rosendahl, J. Keir, and A. Cameron, "BLINCK-A diagnostic algorithm for skin cancer diagnosis combining clinical features with dermatoscopy findings," Dermatology Practical \& Conceptual 2(2), 55-61 (2012).

14. G. Argenziano, G. Fabbrocini, P. Carli, V. De Giorgi, E. Sammarco, and M. Delfino, "Epiluminescence microscopy for the diagnosis of doubtful melanocytic skin lesions," Archives of Dermatology 134, 1563-1570 (1998).

15. I. Bratchenko, V. Sherendak, O. Myakinin, D. Artemyev, A. Moryatov, E. Borisova, L. Avramov, L. Zherdeva, A. Orlov, S. Kozlov, and V. Zakharov, "In vivo hyperspectral imaging of skin malignant and benign tumors in visible spectrum," Journal of Biomedical Photonics \& Engineering 4(1), 010301 (2018).

16. I. Diebele, I. Kuzmina, A. Lihachev, J. Kapostinsh, A. Derjabo, L. Valeine, and J. Spigulis, "Clinical evaluation of melanomas and common nevi by spectral imaging," Biomedical Optics Express 3(3), 467-472 (2012).

17. A. Machihin, V. Batshev, and V. Pozhar, "Aberration analysis of AOTF-based spectral imaging systems," Journal of the Optical Society of America A 34(7), 1109-1113 (2017).

18. I. A. Bratchenko, M. V. Alonova, O. O. Myakinin, A. A. Moryatov, S. V. Kozlov, and V. P. Zakharov, "Hyperspectral visualization of skin pathologies in visible region," Computer Optics 40(2), 240-248 (2016).

19. L. A. Zherdeva, I. A. Bratchenko, O. O. Myakinin, A. A. Moryatov, S. V. Kozlov, and V. P. Zakharov, "In vivo hyperspectral imaging and differentiation of skin cancer," Proceedings of SPIE 10024, 100244G (2015).

20. R. O. Duda, P. E. Hart, and D. G. Stork, Pattern Classification, Wiley (2001).

21. K. Hajian-Tilaki, "Receiver Operating Characteristic (ROC) Curve Analysis for Medical Diagnostic Test Evaluation," Caspian Journal of Internal Medicine 4(2), 627-635 (2013).

22. M. Brenner, V. J. Hearing, "The Protective Role of Melanin Against UV Damage in Human Skin," Photochemistry and Photobiology 84(3), 539-549 (2008).

23. N. S. Eikje, K. Aizawa, and Y. Ozaki, "Vibrational spectroscopy for molecular characterisation and diagnosis of benign, premalignant and malignant skin tumours," Biotechnology Annual Review 11, 191-225 (2005).

24. A. Lihachev, A. Derjabo, I. Ferulova, M. Lange, I. Lihacova, and J. Spigulis, "Autofluorescence imaging of basal cell carcinoma by smartphone RGB camera," Journal of Biomedical Optics 20(12), 120502 (2015).

25. L. Lihacova, K. Bolocko, and A. Lihachev, "Semi-automated non-invasive diagnostics method for melanoma differentiation from nevi and pigmented basal cell carcinomas," Proceedings of SPIE 10592, 1059206 (2017).

26. T. Nagaoka, A. Nakamura, H. Okutani, Y. Kiyohara, H. Koga, T. Saida, and T. Sota, "Hyperspectroscopic screening of melanoma on acral volar skin," Skin Research \& Technology 19(1), e290-e296 (2013).

27. A. Esteva, B. Kuprel, R. A. Novoa, J. Ko, S. M. Swetter, H. M. Blau, and S. Thrun, "Dermatologist-level classification of skin cancer with deep neural networks," Nature 542, 115-118 (2017).

\section{Introduction}

Cancer is one of the most dangerous and yearly progressing diseases. According to the International Agency for Research on Cancer, about 14.1 million new cases of cancer have been detected throughout the world in 2012, and the estimated increase of new cancers by 2025 is up to 19.3 million cases due to the growth of the population and the increase in its average age [1]. Skin cancer is one of the most common forms of cancer [2]. For example, basal cell carcinoma of the skin is the most common type of cancer in the world and accounts for about $75 \%$ of all malignant skin formations.
Melanoma, on the contrary, has the lowest incidence rate in the world ( $9 \%$ ) but ranks first in mortality $(75 \%)$ among all forms of skin cancer [3].

Similar trends in cancer morbidity are also observed in Russia. For example, according to the Moscow Scientific Research Institute of Oncology named after P.A. Hertsen, the incidence of malignant neoplasms in Russia in 2015 was 589341 cases. One of the main localizations in the general (both sexes) structure of the oncological morbidity is skin $(14.2 \%)$ - the second most common oncological pathology in women and third in men [4]. The increase in the incidence of malignant skin 
tumors from 2005 to 2015 is $31.47 \%$, including a $34.84 \%$ increase in melanoma cases.

Taking into account the superficial localization of skin tumors the most convenient way of their early detection is a preventive examination. In the early detection of a tumor, the patient has a higher chance of recovery [5]. However, a visual examination of melanoma at an early stage is usually not enough, the accuracy of diagnosis of pigmented skin tumors in the early stage (especially for first-hand specialists) does not exceed $50 \%$ [4] due to the similarity of melanoma with a nevus.

Different types of skin biopsy do not apply since they are time-consuming and may lead to cosmetic injury [5]. In this regard, studies of many scientific groups are aimed at developing precisely non-invasive methods for primary diagnosis of skin oncological pathologies. The diagnostic efficiency of such methods as CT and MRI falls dramatically for small diameter tumors (less than $1.5 \mathrm{~mm}$ ) due to insufficient spatial resolution [6]. In such cases, a more accurate assessment of the malignancy of skin formations can be obtained with the use of optical diagnostic methods, such as digital dermatoscopy [7], Raman spectroscopy $[8,9]$ and hyperspectral imaging [10].

Dermatoscopy provides the opportunity to enlarge the standard ABCD rule [11] or 7-point checklist [12] and involve additional criteria in suspicious skin tissues inspection. Thus, dermatoscopy opens up a new dimension on clinical morphology of pigmented skin lesions and enables well-trained physicians to improve their diagnostic accuracy up to $65 \%$ [13]. As this accuracy may be obtained only by a well-trained physician, hyperspectral imaging (HSI) technique may be used. This technique simultaneously allows for imaging of the pathology area and spectral data acquiring. The tissue image allows one to estimate tissue morphology and allows for finding the stage of tumor growth, while the spectral data allows one to make a conclusion about the chemical composition of the tested tissue area. For example, the quantitative change of melanin and hemoglobin indicates a high risk of tissue malignancy and leads to a discoloration of the skin, changes of the formation shape and appearance of atypical vascular and pigmentary patterns [14].

In current in vivo clinical trials, we tried to estimate applicability of integrated optical density indexes introduced in our previous preliminary studies [13] and calculated during HSI application in the visible wavelength range $(450-750 \mathrm{~nm})$ for areas of melanin and hemoglobin absorption. Analysis of optical indexes was performed for only neoplasm spectra and neoplasms and adjacent normal skin spectra to estimate the potential of normal skin examination during the neoplasm diagnosis. In contrast to earliest investigations on a smaller cohort of malignant neoplasms [15, 16] (less than 20 malignant tumors) our trial was performed on a cohort of 91 skin neoplasms, including 41 malignant tumors. It helped us to achieve reliable data about integral optical density indexes application in clinical examination of skin cancers. The proposed approach was used to differentiate various skin malignant tumors from the benign. Malignant Melanoma (MM) and basal cell carcinoma (BCC) were studied in the malignant tumors group; in the benign diseases (Benign - BN) group keratomas, hemangiomas and nevi were studied.

\section{Materials and methods}

\subsection{Experimental setup and spectra acquisition}

To register the spectral images of skin we used an acousto-optical (AO) video spectrometer [17], it is structural diagram is shown in Fig. 1.

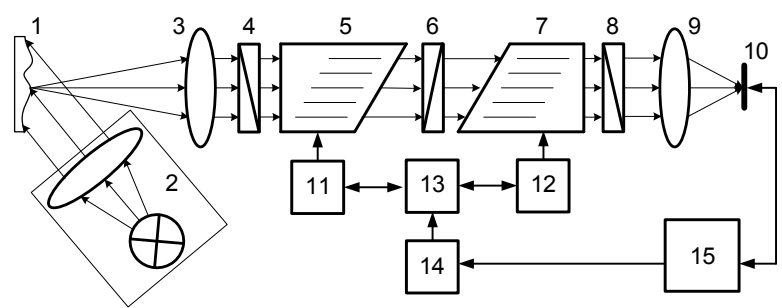

Fig. 1 Registration of skin hyperspectral images: 1 - skin sample, 2 - LED diode, 3, 9 - collection lenses, 5, 7 - AO cells, 4, 6, 8 - polarizers, 10 - camera, 11,12 - high-frequency amplifiers 13 -high-frequency generator, 14 - controller, $15-\mathrm{PC}$.

The broadband radiation of the LED source 2 reflected and scattered from the skin sample 1 is converted by an input lens 3 into a collimated beam and directed to the AO monochromator consisting of two AO cells 5 and 7 and three polarizers: an input 4, an intermediate 6 and output 8. A special feature of AO monochromator is double sequential filtering. It provides almost complete compensation of spatial and spectral image distortions caused by Bragg diffraction in a single anisotropic AO cell [15]. In the proposed scheme two identical AO cells 5 and 7 perform diffraction of ordinary and extraordinary waves. The second cell 7 is rotated around the first AO cell 5 in the diffraction plane by $180^{\circ}$. The polarization plane of the polarizer 6 is orthogonal to the plane of polarization of the identical polarizers 4 and 8 . Light in AO cell 5 diffracts on the dynamic grating produced by the acoustic wave of a given frequency. As a result of diffraction, the radiation changes its linear polarization from the ordinary polarization to the extraordinary one. Similarly, repeated diffraction in the AO cell 7 occurs, which increases the contrast of the isolated spectral component with full compensation of linear and nonlinear distortion. Then, with the aid of the objective 9 , the filtered light is focused on the matrix of the monochrome video camera 10 . By varying the frequency of the acoustic wave the piezoelectrical excitation of the AO cells 5 and 7 occurs, and it is possible to reconstruct the diffraction grating period simultaneously in both AO cells. Thus it is possible to 
Table 1 Summary of patients with skin tumors.

\begin{tabular}{cccc|cccc}
\hline \multirow{2}{*}{ Lesion } & \multirow{2}{*}{ Age } & Male & Female & $\begin{array}{c}\text { chest/ } \\
\text { stomach }\end{array}$ & $\begin{array}{c}\text { Lockation } \\
\text { head }\end{array}$ & limb & back \\
\cline { 3 - 9 } & $38-78$ & 7 & 15 & 2 & 3 & 8 & 9 \\
\hline MM & $37-85$ & 8 & 11 & 3 & 9 & 3 & 4 \\
\hline BCC & $22-82$ & 19 & 31 & 8 & 11 & 14 & 17 \\
\hline BN & 22 & & & &
\end{tabular}

obtain the image of the object 1 at an arbitrary predetermined wavelength $\lambda$ in the range $440-750 \mathrm{~nm}$ with a sufficiently high spectral resolution $(\delta \lambda=2.5 \mathrm{~nm}$ at $\lambda=633 \mathrm{~nm}$ ) and spatial resolution of $0.14 \mathrm{~mm}$.

\subsection{Tissue samples}

There were 91 patients with skin cancers ( 57 females and 34 males, all white, Caucasian, skin phototype I and II) enrolled in this study. A total of $19 \mathrm{BCC}, 22 \mathrm{MM}$ and $50 \mathrm{BN}$ neoplasms (including 16 nevi, 5 papillomas, 4 hemangiomas, 7 different inflammatory diseases, 12 keratomas, 1 fibroma, 1 Bowen disease, 4 keratopapillomas) were studied. The detailed distribution of human skin lesions, including information about tumor locations, is provided in Table 1. Each neoplasm was examined by the oncodermatologist to get a medical specialist diagnosis. Every tumor study was accompanied by histological analysis to make a final diagnosis. The protocols of in vivo tissue diagnostics were approved by the ethical committee of Samara State Medical University. The Institutional Review Board of Samara National Research University approved the study protocols. This research adhered to the tenets set forth in the Declaration of Helsinki. Informed consent of each subject was obtained.

\subsection{Spectra processing and data analysis}

Typical normalized spectra and tumor images stabilization procedure may be found elsewhere [18, 19]. Generally, to obtain quantitative estimations of various human skin tissues backscattered spectra we used data about the spectral optical density of skin tissues. The diffuse backscattered reflectance from the skin in spatial point $(x, y)$ may be characterized by optical density (OD) on the selected wavelength $\lambda$ as:

$$
O D(x, y, \lambda)=\lg \frac{I_{o}(x, y, \lambda)}{I(x, y, \lambda)}
$$

where $I_{0}(x, y, \lambda)$ is backscatter intensity from the background of the light source, $I(x, y, \lambda)$ is backscattered intensity from the tissue sample. While the exact determination of tumor type may be performed with OD analysis based on above spectral bands:

$$
O D_{i}(x, y)=\frac{1}{\lambda_{2}-\lambda_{1}} \int_{\lambda_{1}}^{\lambda_{2}} O D(x, y, \lambda) d \lambda
$$

where hemoglobin-related optical density $O D_{H}$ defined for $\left(\lambda_{1}=530 \mathrm{~nm}, \lambda_{2}=570 \mathrm{~nm}\right)$ and melanin-related $O D_{M}$ - for $\left(\lambda_{1}=600 \mathrm{~nm}, \lambda_{2}=670 \mathrm{~nm}\right)$. Further, we calculated mean $O D_{H}$ and $O D_{M}$ values for all studied tumors and healthy skin. Allocation of tumor area was performed on the basis of the approach presented in our previous studies and was performed based on image segmentation by Otsu method [19]. Healthy skin region was chosen near the tumor area with $1.5-2 \mathrm{~cm}$ indentation from the tumor and included approximately $1 \times 1 \mathrm{~cm}$ area of healthy skin. Example of the hyperspectral image with tumor and healthy skin areas allocation as well as the distribution of $O D_{M}$ coefficient may be found elsewhere [15].

Discrimination of tumors was performed based on two principles. The first principle took into account only raw values of $O D_{H}$ and $O D_{M}$ coefficients, while the second principle used data for the normal skin of the same patient for $O D^{N}{ }_{H}$ and $O D^{N}{ }_{M}$ coefficient as follows:

$$
O D_{i}^{N}=\frac{O D_{i}^{T}}{O D_{i}^{H}}
$$

where $O D^{T}{ }_{i}$ and $O D^{H}{ }_{i}$ are $O D_{i}$ coefficients calculated for one patient for tumor (index ${ }^{T}$ ) and healthy skin (index ${ }^{H}$ ) areas respectively.

Finally, we analyzed integral properties of skin for allocated tumor area and healthy skin area with an unbiased dispersion of $O D_{i}$ and $O D^{N}{ }_{i}$ coefficients inside the area of interest:

$$
\overline{O D}_{i}=\frac{1}{N-1} \sum_{n=1}^{N}\left(O D_{n}-\bar{M}\right)^{2}
$$

where $N$ is the number of pixels in the area of interest, $O D_{n}$ is the OD (or normalized OD) in the $n^{\text {th }}$ pixel of the area of interest, $\bar{M}$ is the mean value of $O D_{n}$ inside the area of interest.

In skin tissues classification we utilized $\overline{O D}_{i}$ and $O D^{N}{ }_{i}$ coefficients. The choice of these coefficients was based on coefficients utility. While $O D^{N}{ }_{i}$ coefficients provide information about changes of chromophores content in the tumor in comparison to healthy skin of the same person, $\overline{O D}_{i}$ coefficients provide information 


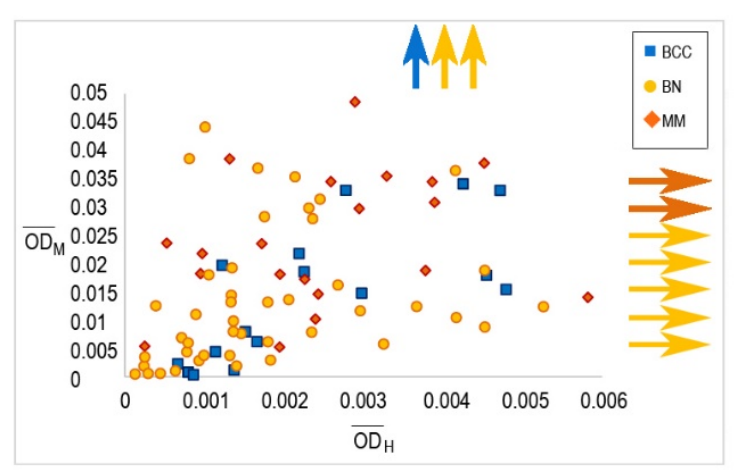

a

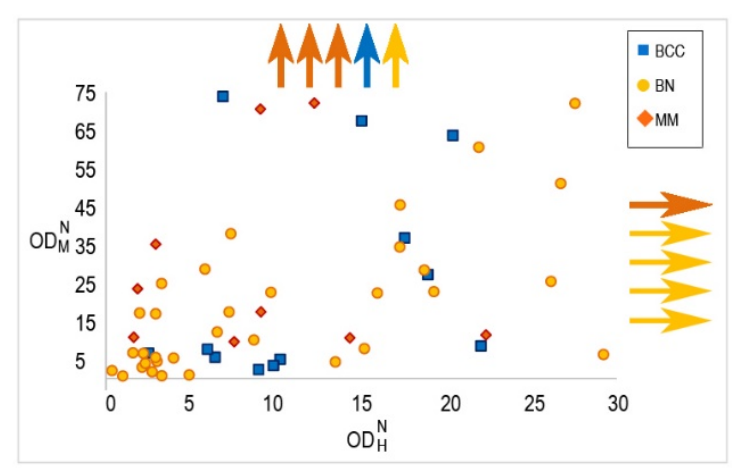

b

Fig. 2 Distribution of skin neoplasms on phase planes with $\overline{O D}_{i}$ (a) and $O D^{N}{ }_{i}$ (b) axes.

Table 2 Accuracy (\%) of neoplasms classification

\begin{tabular}{cccccccc}
\hline Classes & $\overline{O D}_{H}$ & $\overline{O D}_{M}$ & $O D^{N}{ }_{H}$ & $O D_{M}^{N}$ & $\overline{O D}_{H}+\overline{O D}_{M}$ & $O D^{N}{ }_{H}+O D^{N}{ }_{M}$ & Physician \\
\hline MM+BCC vs BN & 61 & 71 & 65 & 60 & 76 & 78 & 51 \\
\hline MM vs BCC & 54 & 59 & 66 & 59 & 68 & 79 & 56 \\
\hline MM vs BCC+BN & 60 & 69 & 68 & 63 & 75 & 75 & 51 \\
\hline
\end{tabular}

about mean values of chromophores concentration in the tumor area. Thus, these two approaches use different features of chromophores distributions and both may be useful in skin tissues classification.

$\overline{O D}_{i}$ and $O D^{N}{ }_{i}$ coefficients were used for tissue classification by discriminant analysis (DA). DA can separate two or more classes based on different statistical parameters of Gaussian distributions. The efficiency of the proposed approach is characterized by sensitivity and specificity and ability to select defined classes in different areas of the phase plane. The analysis of skin tissues data allocation was performed using quadratic DA classifiers [20]. In addition, data were analyzed using SPSS software version 23 (SPSS Inc., Chicago, IL, USA) with logistic regression, and a $\mathrm{P}$-value of $<0.01$ was considered to indicate statistically significant differences. Then, the plot of Sensitivity versus 1-Specifity (called receiver operating characteristic (ROC) curve) is built, and the area under the curve (AUC) was used as an effective criterion of skin tissues discrimination accuracy. This curve plays a central role in evaluating diagnostic ability of tests to discriminate the true state of subjects, finding the optimal cut off values, and comparing alternative diagnostic tasks when each task is performed on the same subject [21].

\section{Results and discussion}

Differentiation of skin neoplasms was performed with phase plane analysis. In the separation of phase plane classes axes of the plane are criterions of tissues classification, and every tested sample is a point on the phase plane with coordinates corresponding to the criterion values for this tissue sample. Differentiation of $\mathrm{BN}$ pathologies from malignant neoplasms is possible with backscattered spectrum analysis in the visible region since it contains data on hemoglobin and melanin content in skin tissues. Fig. 2 demonstrates the joint application of $\overline{O D}_{i}$ and $O D^{N}{ }_{i}$ coefficients for skin tissues classification. These distributions may help classify neoplasms based on quadratic DA implementation. For example, the most accurate discrimination of $\mathrm{MM}$ and BCC is possible using 7.8 and 14.3 thresholds of $O D^{N}{ }_{M}$ and $O D^{N}{ }_{H}$ coefficients values. Most efficient malignant and benign tissues separation is possible using 0.016 and 0.0014 thresholds of $\overline{O D}_{M}$ and $\overline{O D}_{H}$ coefficients values. In addition, general information about neoplasms classification accuracy with proposed coefficients and oncological specialist collected in Table 2.

Analysis of Fig. 2 and Table 2 helps to reveal possibilities of HSI integral information application in skin neoplasms classification. Application of single $\overline{O D}_{i}$ or $O D^{N}{ }_{i}$ coefficient for melanin content leads to $60 \%-70 \%$ accuracy and these coefficients for hemoglobin content provides a slightly lower accuracy of $55 \%-65 \%$. It is important to note, that accounting of normal healthy skin helps to improve accuracy for $\overline{O D}_{H}$ and decreases accuracy for $\overline{O D}_{M}$. These facts may be caused by inhomogeneity of melanin and hemoglobin distribution in the skin. While neoplasms have a heterogeneous distribution of hemoglobin and capillary network, normal skin has more homogeneous one [20], 
and, thus, comparison of $\overline{O D}_{H}$ dispersion in neoplasm and normal tissue provides additional information for tissues discrimination. Melanin content of neoplasms frequently may be increased in comparison to healthy skin, but as every person have its own skin phenotype and unique level of melanin [22] it may be complicated to estimate differences in melanin content of neoplasm and healthy skin. That is why most likely in our study application of $O D^{N}{ }_{M}$ coefficient showed a rather low accuracy of skin neoplasms classification in comparison to $\overline{O D}_{M}$ comparison.

Application of coefficients combination $\left(\overline{O D}_{H}+\overline{O D}_{M}\right.$ and $O D^{N}{ }_{H}+O D^{N}{ }_{M}$ ) helps to improve the accuracy of tissues classification by $5 \%-15 \%$ in comparison with single coefficient implementation. $O D^{N}{ }_{H}+O D^{N}{ }_{M}$ combination in comparison with $\overline{O D}_{H}+\overline{O D}_{M}$ pair shows almost the same rates of accuracy for cases of MM vs other neoplasms and malignant neoplasms vs BN classification but demonstrates $11 \%$ higher accuracy in case of MM vs BCC classification. That may be explained by $O D_{H}^{N}$ coefficient usage as it shows high rates of tissues classification in comparison with unnormalized on healthy skin $\overline{O D}_{H}$ coefficient. Thus, we may try to combine normalized and unnormalized coefficients to achieve the highest performance of tissues classification.

In order to do this, we performed ROC analysis for the combination of proposed coefficients. Fig. 3 demonstrates ROC curves for different pairs of coefficients. One may see that that the best AUC values shows a combination of normalized and unnormalized coefficients. This fact proves that the best performance of HSI skin neoplasms study may be implemented with a joint analysis of pathology area and healthy skin. Combination of $O D^{N}{ }_{H}+\overline{O D}_{M}$ and $\overline{O D}_{H}+\overline{O D}_{M}$ coefficients shows 0.693 and 0.686 AUC values respectively, while $O D^{N}{ }_{H}+O D^{N}{ }_{M}$ coefficients pair shows only 0.608 values. As higher AUC value the higher final accuracy of classification is, thus, unnormalized and combined approaches of neoplasm hemoglobin and melanin dispersion estimation may be implemented in clinical practice. Moreover, comparison of HSI analysis and physician survey accuracy presented in Table 2 shows that automatic analysis may significantly improve the accuracy of skin neoplasm detection. HSI analysis may be used during the first-hand examinations/ that suffer from very low specificity of malignant tumors detection [23].

Considering alternative optical methods of the proposed approach, one may say that the most frequently used today method is multispectral analysis with additional spectral ranges or native fluorescence analysis. For example, it was proposed to use the spectral region up to $1000 \mathrm{~nm}$ and analyze the content of NADH/ NAD+ $[24,25]$. The total sensitivity and specificity for separation of MM from nevus and BCC were $97 \%$ and $96 \%$ respectively. However, this approach requires an additional light source (either a separate source for the near-IR or a white diode with a near-IR coating up to $1000 \mathrm{~nm}$ ), as well as a camera with a higher sensitivity (in comparison with $\mathrm{CCD}$ ) in the 1000-1100 range $\mathrm{nm}$, which significantly complicates the design of the multispectral camera. In the study by Nagaoka et al. [26] an index is proposed that describes the heterogeneity of the tumor, which is the analog of the color index in dermatoscopy. The sensitivity of the determination of melanoma was $90 \%$, and specificity was $84 \%$. However, it is important to note that described above results [24-26] were obtained on a small number of samples (only five-ten melanomas were included in the studied cohort).

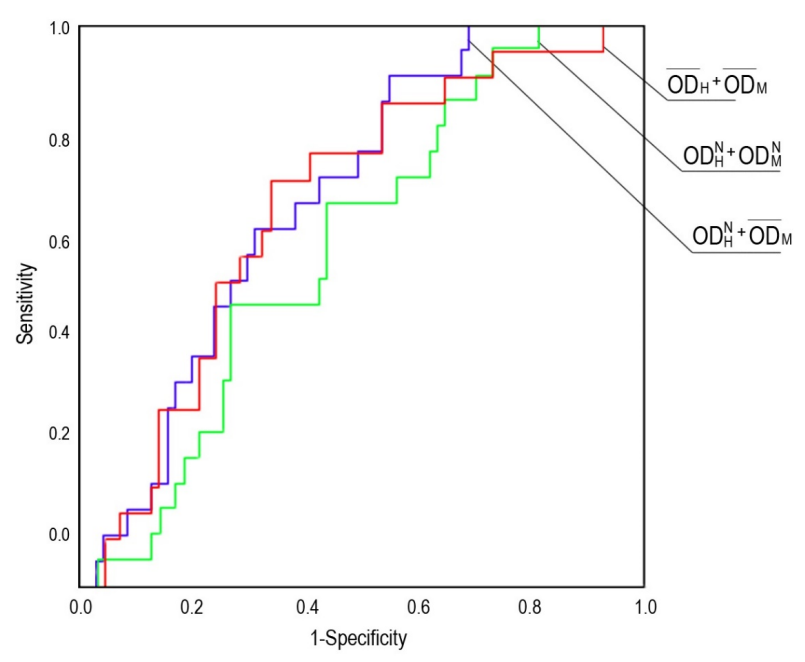

Fig. 3 ROC curves of MM vs $\mathrm{BCC}+\mathrm{BN}$ classification with logistical regression for $O D^{N}{ }_{H}+\overline{O D}_{M}, O D^{N}{ }_{H}+O D^{N}{ }_{M}$ and $\overline{O D}_{H}+\overline{O D}_{M}$ coefficients.

Evolution of the proposed technique may be based on the approach of spatial evaluation of diagnostic dermatoscopic signs, and for example, may be implemented with convolutional neural networks analysis [27]. The authors in Ref. [27] claimed maximum sensitivity and specificity of melanoma diagnosis at $90 \%$ and $85 \%$, respectively. Therefore, image analysis approaches may be combined with the proposed technique to improve the overall accuracy in HSI skin cancer imaging.

\section{Conclusions}

The proposed approaches to skin tissues classification showed $68 \%-78 \%$ accuracy. These values of accuracy are not quite high in comparison to $90 \%$ and higher accuracies of such optical approaches as Raman or fluorescent spectroscopies [8]. However, integral information about skin chromophores may significantly increase the accuracy of visual inspection by a dermatologist, as the proposed technique demonstrated $15 \%-20 \%$ higher accuracy in skin tumors classification. Wherein application of both $\overline{O D}_{i}$ and $O D^{N}{ }_{i}$ coefficients and their combination leads to the comparable accuracy in tumors classification. This fact proves that choice of visible range area $(450-750 \mathrm{~nm})$ for the analysis of the 
chemical composition of skin may be useful in oncodermatological applications.

It should be noted, that in the proposed approach the processing of hyperspectral data is carried out in real time and does not require operator participation. In general, the simplicity of the equipment and the speed of processing results may allow for adapting the proposed method for tasks of mass screening of skin tumors. Moreover, in future works on the development of the proposed approach, we may increase the number of examined skin neoplasms samples and also combine the proposed technique with image pattern analysis methods.

\section{Disclosures}

All authors declare no conflict of interests for this manuscript and have no a financial interest in the materials used in the manuscript.

\section{Acknowledgments}

This research was supported by RFBR grant № 17-3850145 and the Ministry of Education and Science of the Russian Federation. 